\title{
tlpA gene expression is required for arginine and bicarbonate chemotaxis in Helicobacter pylori
}

\author{
Oscar A. Cerda\#, Felipe Núñez-Villena*, Sarita E. Soto*, José Manuel Ugalde*, Remigio López-Solís* \\ and Héctor Toledo*\& \\ \# Department of Neurobiology, Physiology and Behavior, University of California, Davis, CA 95616-8519 \\ * Laboratorio de Microbiología Molecular, Programa de Biología Celular y Molecular, ICBM, Facultad de Medicina, Universidad de Chile. Avenida Independencia 1027. \\ Casilla 70086, Santiago-7, Chile
}

\begin{abstract}
About half of the human population is infected with Helicobacter pylori, a bacterium causing gastritis, peptic ulcer and progression to gastric cancer. Chemotaxis and flagellar motility are required for colonization and persistence of $H$. pylori in the gastric mucus layer. It is not completely clear which chemical gradients are used by $\mathrm{H}$. pylori to maintain its position. TlpA, a chemotaxis receptor for arginine/ bicarbonate, has been identified. This study aimed to find out whether $\operatorname{tlp} A$ gene expression is required for the chemotactic response to arginine/ bicarbonate. Wild-type motile H. pylori ATCC 700392 and H. pylori ATCC 43504, a strain having an interrupted tlpA gene, were used. Also, a tlpA-knockout mutant of H. pylori 700392 (H. pylori 700-tlpA::cat) was produced by homologous recombination. Expression of $\operatorname{tl} A$ was assessed by a Reverse Transcriptase-Polymerase Chain Reaction (RT-PCR) assay. Chemotaxis was measured as a Relative Chemotaxis Response (RCR) by a modified capillary assay. H. pylori 700392 presented chemotaxis to arginine and sodium bicarbonate. $H$. pylori 700-tlpA::cat showed neither tlpA gene expression nor chemotaxis towards arginine and bicarbonate. Besides confirming that TlpA is a chemotactic receptor for arginine/bicarbonate in $H$. pylori, this study showed that $t$ lpA gene expression is required for arginine/bicarbonate chemotaxis.
\end{abstract}

Key words: $\operatorname{tl} A$, chemotaxis, Helicobacter pylori, arginine, bicarbonate.

\section{INTRODUCTION}

Helicobacter pylori, a motile Gram-negative human pathogen that causes gastritis and duodenal/gastric ulcers and represents a high risk of gastric cancer, inhabits the gastric mucus layer (McGowan et al., 1996). Most of these bacteria live deep in the layer of mucus gel and close to the surface of the epithelium. Mucus is continuously secreted by surface epithelial cells of the gastric glands and is degraded at the luminal surface of the mucus layer (Schreiber and Scheid, 1997). Because of a rapid mucus turnover, H. pylori cells need motility and spatial orientation to avoid being dragged into the lumen, where the acidic $\mathrm{pH}$ inhibits growth and paralyzes cell motility (Schreiber et al., 1999; Worku et al., 1999). Accordingly, orientation plays a central role both in acute colonization and chronic persistence of $H$. pylori.

Motile bacteria sense chemical gradients by means of chemoreceptor proteins that relay the information to the flagellar motor (Bren and Eisenbach, 2000). All gastric Helicobacter species are highly motile. In recent years, comparative genomics in various Helicobacter species and related bacteria has facilitated the analysis of genes. Experiments with $H$. pylori in different animal models have shown that flagellar motility is essential to colonize the gastric mucusa (Ernst and Gold, 2000). H. pylori shows taxis response towards urea, amino acids and bicarbonate whereas it moves away from $\mathrm{H}^{+}$(Cerda et al., 2003; Croxen et al., 2006; Mizote et al., 1997; Worku et al., 2004). In addition to motility, recent studies in in vivo systems have shown that $H$. pylori chemotaxis is required for colonization and inflammatory response induction in gastric mucosa (Andermann et al.,
2002; Williams et al., 2007). However, it is still unclear which combination of chemical gradients $H$. pylori uses in vivo to maintain an optimal position in the gastric mucus layer (Schreiber et al., 2004). By using genomic analysis it has been shown that the chemotaxis system of $H$. pylori is genetically similar to the one in Salmonella. However, extensive functional analysis of potentially participating proteins is still necessary. Only four genes with homology to chemotaxis receptors have been identified in H. pylori: $\operatorname{tl} A, t \operatorname{lp} B, t \operatorname{lpC}, t \operatorname{lp} D$ (Tomb et al., 1997). Sensing specificities of these four annotated $H$. pylori chemosensors have not been comprehensively described. In vitro negative taxis to acidic $\mathrm{pH}$ was found to be dependent on the sensor protein TlpB (Croxen et al., 2006). On the other hand, Schweinitzer et al. (2008) reported that TlpD is a receptor for energy taxis. Positive taxis to arginine and bicarbonate have been observed in vitro (Cerda et al., 2003; Mizote et al., 1997; Worku et al., 2004) and reported to be dependent on TlpA function (HP0099, according to the annotated genome sequence of $H$. pylori strain 26695) (Cerda et al., 2003). The H. pylori sensor TlpA has been expressed heterologously in E. coli and found to provide tactic movement towards arginine, bicarbonate and urea (Cerda et al., 2003). Interestingly, the $\operatorname{tlp} A$ gene was found to be interrupted by a mini IS605 sequence in the H. pylori 43504 strain, which fails to recognize either arginine or sodium bicarbonate as chemoattractants (Cerda et al., 2003). However, straindependency has not been discarded yet. In this work, we present further evidence on the role of TlpA as a chemotactic receptor by showing that $t l p A$ disruption in the $H$. pylori wildtype strain ATCC 700392 causes loss of in vitro chemotactic response to arginine and bicarbonate. 


\section{MATERIALS AND METHODS}

H. pylori strains

Bacterial strains used in this study were $H$. pylori strains ATCC 700392 and ATCC 43504. In addition, in this study $H$. pylori 700 tlpA::cat was developed. Frozen stocks and replated cultures of the $H$. pylori strains were used. As recommended by ATCC, the strains were cultivated on TSA agar plates [trypticase soy agar plates (Becton Dickinson Biosciences) supplemented with 5\% sheep blood (Public Health Institute of Chile), culture supplement Vitox (Oxoid) and antibiotic culture supplement Dent (Oxoid)] for $24 \mathrm{~h}$ at $37{ }^{\circ} \mathrm{C}$ in $5.5 \% \mathrm{CO}_{2}$ and $85 \%$ humidity.

\section{Chemotaxis assay}

Bacterial cells were scraped from the plates and suspended in chemotaxis buffer $(10 \mathrm{mM}$ potassium phosphate, $\mathrm{pH}$ $7.0 ; 3.0 \%$ polyvinylpyrrolidone) at a concentration of 3.0 $x 10^{8}$ cells per $\mathrm{ml}\left(\mathrm{OD}_{560}=0.4\right)$. The chemotaxis assay was done as previously described by Cerda et al. (2003). Briefly, $100 \mu 1$ of bacterial suspension were placed into a $200-\mu 1$ disposable pipette tip. On the other hand, a $100 \mu \mathrm{l}$ volume of a solution containing $10 \mathrm{mM}$ of the compound to be tested for chemotactic response (buffer alone served as control) was aspirated through a $25 \mathrm{G}$ stainless-steel needle $(0.254 \mathrm{~mm}$ ID x $20 \mathrm{~mm}$ long) into a 1-ml tuberculin syringe. The needlesyringe system was fitted to the pipette tip in such a way that most of the needle became immersed into the bacterial suspension. The system was positioned horizontally and incubated at $30{ }^{\circ} \mathrm{C}$ for $45 \mathrm{~min}$. Finally, the needle-syringe system was separated from the bacterial suspension, cleaned externally and 10-fold serially diluted in chemotaxis buffer. Dilutions were plated onto $4 \%(\mathrm{w} / \mathrm{v})$ trypticase soy agar plates supplemented with 5\% horse serum (HyClone), culture supplement Vitox (Oxoid) and antibiotic culture supplement Dent with 5.5\% $\mathrm{CO}_{2}$ and $85 \%$ humidity. Those culture conditions enhanced visualization of colonies. After $24 \mathrm{~h}$ incubation at $37{ }^{\circ} \mathrm{C}$ the number of colony-forming units (CFUs) per plate was counted. Each assay was performed in duplicate. Results were expressed as the mean of at least five independent assays. To ascertain whether a test compound was or was not an attractant, a relative chemotaxis response (RCR) was calculated as the ratio between the number of bacteria entering the needle-syringe system in a dilution dependent manner and the number of bacteria in the control condition. A relative chemotaxis response of 2 or greater was considered significant (Adler, 1973; Cerda et al., 2003; Mazumder et al., 1999; Moulton and Montie, 1979). Differences between groups were analyzed statistically by using the Student's t-test.

\section{Motility assay}

Bacterial cells grown in $5.5 \% \mathrm{CO}_{2}$ and $85 \%$ humidity at $37{ }^{\circ} \mathrm{C}$ for 5 days on TSA agar plates were scrapped and suspended in phosphate saline buffer $\mathrm{pH} 7.2$ (PBS). The suspended cells were stab inoculated with toothpicks into plates containing 0.3\% agar (Difco), trypticase soy broth (Becton Dickinson Biosciences), 5\% horse serum (HyClone), culture supplement Vitox and antibiotic culture supplement Dent. Cells were cultured at $37{ }^{\circ} \mathrm{C}$ for $48 \mathrm{~h}$ in $5.5 \% \mathrm{CO}_{2}$ and $85 \%$ humidity. Motility was scored by measuring the diameter of the growth zone after $48 \mathrm{~h}$ (Cerda et al., 2003).

\section{DNA manipulations and genetic techniques}

Chromosomal DNA from H. pylori was isolated as previously described (Owen and Bickley, 1997). To produce a tlpA knockout $H$. pylori mutant, a PCR $\operatorname{tlp} A$ amplicon from $H$. pylori strain 700392 (Cerda et al., 2003) was firstly cloned into pBR322. Then, the chloramphenicol acetyl transferase gene (cat) from C. coli (Wang and Taylor, 1990) was inserted at a $S a c I$ restriction site of $t l p A$ to create the plasmid pBR322tlpA::cat. Log phase recipient cells were prepared from overnight TSA agar plates. To do so, bacteria were scraped from the agar surface, washed twice in $1 \mathrm{ml}$ of $10 \%$ cold glycerol and recovered after spun down at $2935 \mathrm{xg}$ for $6 \mathrm{~min}$ in an Eppendorf centrifuge 5415C. The bacterial sediment was resuspended in $0.5 \mathrm{ml}$ of $10 \%$ glycerol, mixed with $3-8$ $\mu \mathrm{g}$ of pBR322-tlpA::cat plasmid DNA and the suspension was spotted onto bacterial TSA agar plates followed by incubation for $12-16 \mathrm{~h}$ in $5.5 \% \mathrm{CO}_{2}$ and $85 \%$ humidity to enhance transformation. Bacteria were scrapped from the agar surface and suspended in a minimal volume of PBS to inoculate TSA agar plates containing $15 \mu \mathrm{g} \mathrm{ml}^{-1}$ of chloramphenicol. Transformed colonies (H. pylori 700-tlpA::cat) were isolated from the plates after incubation for 4-5 days. Further details of the procedure for insertion mutation were obtained from Croxen et al. (2006) and Andermann et al. (2002). Correct allelic replacement was confirmed by PCR of genomic DNA isolated from resistant colonies, using TlpA-F and TlpA-R primers (Table 1). Treatments of DNA with restriction enzymes, T4 DNA ligase and T4 DNA polymerase were performed according to protocols recommended by the supplier (Promega).

\section{$m R N A$ extraction and $R T-P C R$ analysis}

Total mRNA from $H$. pylori 700395, H. pylori 43504 and the $H$. pylori 700-tlpA::cat mutant were isolated and purified using RNeasy Mini Kit (Qiagen). Total cDNA was synthesized using cDNA CoreKit (Bioline) following manufacturer's instructions. PCRs were performed in a PTC-100 MJ Research thermal cycler using cTlpA-F and cTlpA-R primers and 16S-F and $16 \mathrm{~S}-\mathrm{R}$ as internal control (16S rDNA H. pylori-specific primers) (Table 1).

\section{RESULTS AND DISCUSSION}

Metabolic reconstitution experiments based on genomics data of $H$. pylori showed the essential character of at least eight amino acids (i.e. alanine, arginine, histidine, leucine, methionine, phenylalanine, valine and cysteine) in the absence of sulphate as sulfur source (Schilling et al., 2002). Against this background, we tested the chemotactic response of the H. pylori 43504 and 700392 strains aiming to identify new TlpA ligands. In these experiments, seven of ten tested amino acids proved to be non attractants in both strains. In accordance with previous results (Cerda et al., 2003), both strains recognized L-serine and L-aspartate as attractants. However, L-arginine was attractant for H. pylori 700392 but non attractant for H. pylori 43504 (Fig. 1). 
TABLE 1

Primers used in the study

\begin{tabular}{lll}
\hline \multicolumn{1}{c}{ Primer } & \multicolumn{1}{c}{ Sequence } & \multicolumn{1}{c}{ Reference } \\
\hline TlpA-F & 5' CGATTGGACGTCTTTTTAATCC 3' & Cerda et al, 2003 \\
TlpA-R & 5' CCCGCAAAAGCTTCTTTAGC 3 & Cerda et al, 2003 \\
TlpB-F & 5' CCGCATATGATGTTTTCTTCAATGTTTGC 3' & This study \\
TlpB-R & 5' CCGGGATCCATTAAAACACGCCGTGATCAC 3' & This study \\
TlpC-F & 5' ATG AAA TC TACA AGA ATT GG 3' & This study \\
TlpC-R & 5' TTC TTT TAA GGT AAT AGA GG 3' & This study \\
16S-F & 5'GCTAAGAGATCAGCCTAT 3' & This study \\
16S-R & 5'CCTACCTCTCCCACACTCTA 3' & This study \\
\hline
\end{tabular}

Previously, we had found that tlpA (ORF HP0099) codes for a receptor protein that recognizes arginine and sodium bicarbonate as attractants in $H$. pylori 700392 . In addition, we found that the lack of chemotactic behavior of $H$. pylori 43504 strain towards arginine and bicarbonate was associated with a mini-IS605 insertion in the $\operatorname{tlp} A$ gene. This observation provided a knockout model for the TlpA function. In order to confirm that the loss-of-function of the $\operatorname{tlp} A$ gene in the $H$. pylori 43504 strain was not a strain-dependent phenomenon we assayed the effect of disrupting the $\operatorname{tlp} A$ gene in $H$. pylori 700392. This strain is chemotactic to arginine/bicarbonate. To this end, we inserted a cat cassette into the $\operatorname{tlp} A$ gene (Fig. 2A). Insertion into tlp $A$ was confirmed by PCR amplification and observation of either the expected $\sim 2 \mathrm{~kb}, 2.3 \mathrm{~kb}$ or $3 \mathrm{~kb}$ bands in H. pylori 700392, H. pylori 43504 and H. pylori 700-tlpA::cat mutant, respectively (Fig. 2B). No differences in amplicon size were observed in the MCPs genes tlpB (ORF HP0103) and

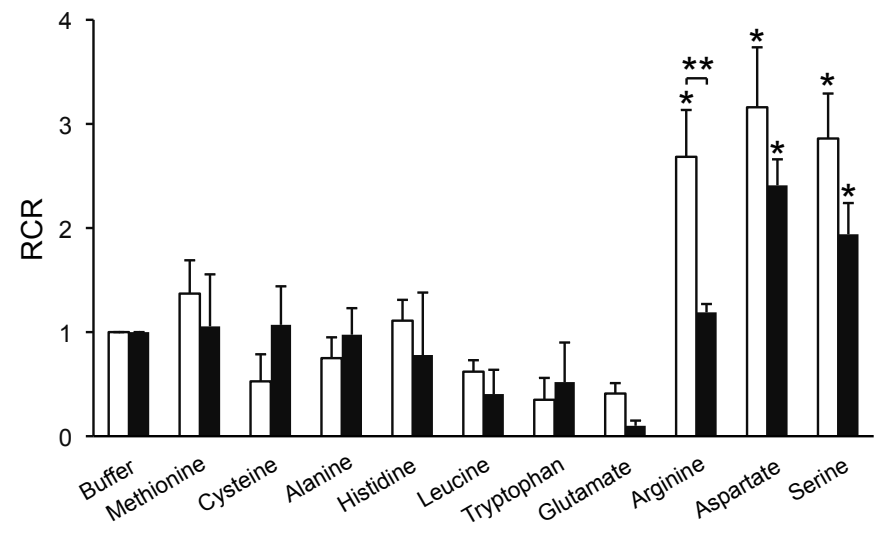

Figure 1. H. pylori 700392 is attracted by aspartate, serine and arginine. Relative chemotactic response (RCR) of $H$. pylori 43504 (filled bars) and 700392 (empty bars) to $10 \mathrm{mM}$ amino acids. A buffer solution served as a negative control and $10 \mathrm{mM}$ aspartate and $10 \mathrm{mM}$ serine as positive controls, as described for strain 700392 (Cerda et al., 2003). Chemotactic responses were tested using a capillary assay, as described under Materials and Methods. Each bar represents average and corresponding standard deviation of at least 5 independent experiments $\left({ }^{*} p<0.05,{ }^{* *} p<0.01\right)$.
tlpC (ORF HP0082) from H. pylori 700392, H. pylori 43504 and H. pylori 700-tlpA::cat strains, thus showing a single allelic replacement of the tlpA gene (Fig. 2B).

A

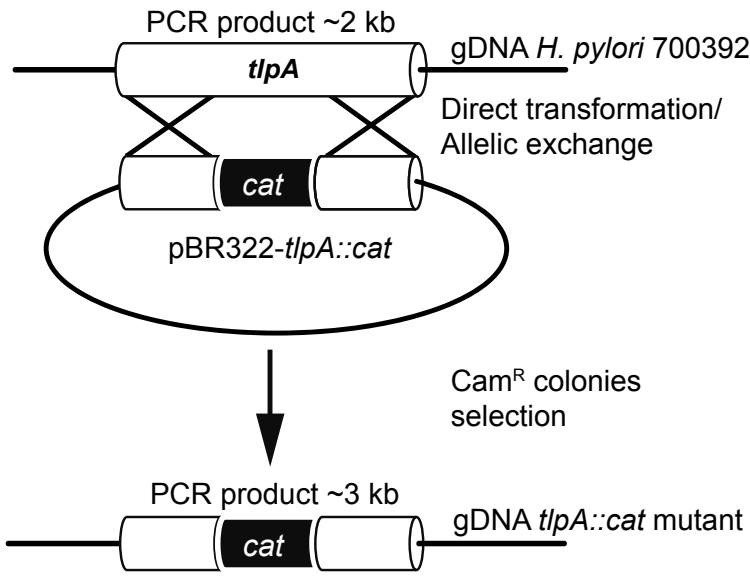

B

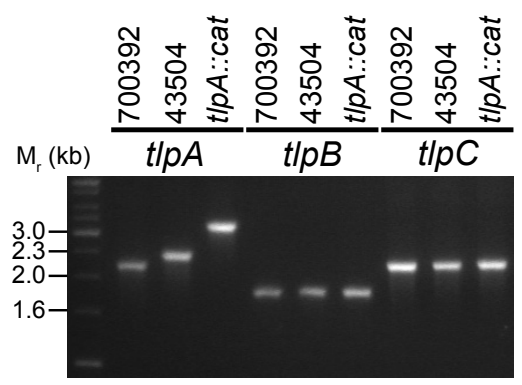

Figure 2. Construction of the tlpA::cat mutant in H. pylori 700392. A. Schematic outline of $H$. pylori 700tlpA::cat mutant construction through the allelic replacement of t/pA gene in $H$. pylori 700392. Predicted $\mathrm{PCR}$ amplicons with TIPA-F/TlpA-R primers from genomic DNA (gDNA) are shown. B. $t / p A, t / p B$ and $t / p C P C R$ amplification from $H$. pylori 700392, 43504 and 700tlpA::cat gDNA demonstrates the replacement of t/pA gene in $H$. pylori 700tlpA::cat mutant. No differences between $t / p B$ and $t / p C$ amplicon sizes were observed. 
Synthesis of $\operatorname{tlp} A$ mRNA in the H. pylori 700-tlpA::cat mutant was evaluated by RT-PCR. From the analysis of total cDNA, no expression was detected in H. pylori 43504 and $H$. pylori 700-tlpA::cat mutant, thus showing that the mini-IS605 and the cat insertions cause loss of tlpA expression on both $H$ pylori strains (Fig. 3A). Next, the motile behavior was tested as to whether tlpA loss-of-function caused a negative motile phenotype in the bacterium. Soft agar assays showed that the H. pylori 700-tlpA::cat mutant and the H. pylori 43504 and 700392 strains present a similar motility behavior. The diameter of growth halo for the three $H$. pylori strains ranged between 18 and $24 \pm 2 \mathrm{~mm}$ after $48 \mathrm{~h}$ (Fig. 3B), thus demonstrating that the $\operatorname{tlp} A$ insertion mutation in $H$. pylori 700-tlpA::cat does not alter the swimming behavior of the bacteria. Accordingly, we assayed the chemotactic response towards sodium bicarbonate and L-arginine using the H. pylori 700-tlpA::cat mutant. This strain was found to exhibit a similar chemotactic phenotype as that of H. pylori 43504, that is, no chemotactic response either to sodium bicarbonate or arginine (Fig. 4, Table 2). These results confirm our previous conclusion that $\operatorname{tlp} A$ codes for a chemotactic receptor that in $H$. pylori recognizes arginine and bicarbonate as attractants.

Motility and chemotaxis have been considered two important processes in colonization, persistence and inflammatory response (Andermann et al., 2002; Williams et al., 2007; Pittman et al., 2001; Ottemann and Lowenthal, 2002; McGee et al., 2005; Terry et al., 2005; Wunder et al., 2006; Castillo et al., 2008; Lowenthal et al., 2009). Tlps chemotactic receptors constitute a well known group of proteins playing an adaptive role in $H$. pylori. Various authors have described the roles of TlpA, and $\mathrm{TlpB}$ in $H$. pylori colonization and persistence (Croxen et al., 2006; Andermann et al., 2002).

H. pylori niche is the stomach mucus layer in which a $\mathrm{pH}$ gradient is established between lumen ( $\mathrm{pH}$ 3.0) and epithelium ( $\mathrm{pH}$ 7.0). Local $\mathrm{pH}$ variations may represent a limit condition for $H$. pylori chemotaxis in its niche, thus restricting the local stomach colonization (Schreiber et al., 2004). H. pylori infection is predominant in antrum and corpus. Positive taxis towards arginine and bicarbonate could participate in territory preferences of $H$. pylori in stomach colonization. On the other hand, Croxen et al., (2006) demonstrated the role of TlpA in $\mathrm{pH}$ negative taxis and colonization. Urease is the major factor in acid resistance (Mendz and Hazell, 1996). This enzyme hydrolyzes urea to ammonia and carbon dioxide, thus favoring proton neutralization. In addition, bicarbonate secretion by gastric epithelia is related to local $\mathrm{pH}$ neutralization. Bicarbonate is secreted into the gastric mucosa by a chloridebicarbonate exchanger that is localized in parietal cells whereas $\mathrm{Na}+$ is secreted by a $\mathrm{Na}^{+}-\mathrm{H}^{+}$exchanger that is localized in the mucous neck cells, chief cells and surface mucous cells (Stuart-Tilley et al., 1994). The chemotactic response to sodium bicarbonate may also contribute to the persistence of $H$. pylori. Since the bicarbonate anion is one of the reaction products of urease activity, this response might be important in the absence of urea. Arginine uptake may constitute an important survival mechanism of $H$. pylori in the stomach niche. In H. pylori, arginine is both an essential amino acid (Schilling et al., 2002)

TABLE 2

Chemotactic response of $H$. pylori to arginine and bicarbonate

\begin{tabular}{lcc}
\hline Condition & \multicolumn{2}{c}{$\mathrm{N}^{\circ}$ of CFUs* /syringe $($ mean $\pm \mathrm{SD}$ ) at 45 min } \\
\cline { 2 - 3 } & H. pylori 700392 & H. pylori 700 tlp A::cat \\
\hline Buffer & $637 \pm 25$ & $343 \pm 17$ \\
Bicarbonate & $1.400 \pm 38$ & $345 \pm 10$ \\
Arginine & $1.705 \pm 43$ & $296 \pm 20$ \\
\hline
\end{tabular}

$\left.{ }^{*}\right)$ CFUs: colony-forming units.
A

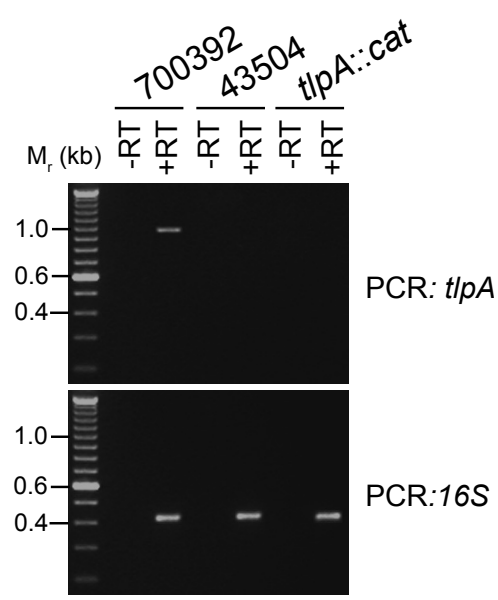

B

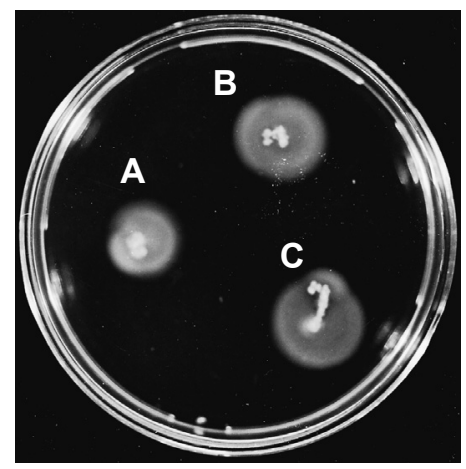

Figure 3. tIpA loss-of-function does not alter motile phenotype in H. pylori 700tlpA::cat mutant. A. tlpA RT-PCR from 700392,43504 and 700tIpA::cat total mRNA. Note the lack of tlpA expression in both H. pylori 43504 and H. pylori 700 tlpA::cat mutant due to mini-IS605 and cat cassette insertions, respectively. No reverse transcriptase in the reaction mix (-RT) with $H$. pylori 700392 mRNAs was used as negative control. B. Motility assays in soft agar. Cell suspensions of H. pylori 700392 (A), H. pylori 43504 (B) and H. pylori 700tlpA::cat (C) were stabbed on $0.3 \%$ agar TSA plates and incubated as described under Materials and Methods. Both mutant strains spread in clear concentric rings because of their motility (representative experiment). 
and a substrate for urea cycle, a metabolic pathway implicated in nitrogen metabolism in this organism (Mendz and Hazell, 1996). Therefore, positive taxis towards arginine could favor its uptake in the gastric environment, thus producing metabolic effects. By both avoiding low $\mathrm{pH}$ zones, as a primary mechanism, and approaching regions of the stomach with high levels of arginine, bicarbonate and other aminoacids, as a secondary one, bacteria could improve their colonization fitness. In this regard, crosstalk signaling between TlpA and TlpB pathways could play a major role in antrum colonization. It is well known that MCPs may form different arrays and organize complex networks between different receptors, in which CheW, CheA, CheR and CheB proteins are involved, thus enhancing signal transduction. Even though in H. pylori CheB/CheR enzymes have not been yet identified, other adaptive proteins may play related roles in this organism. For instance, the $\mathrm{CheV}$ paralogs $\mathrm{CheV1}$, CheV2 and CheV3, which have been proposed as MCPs interacting proteins, have been found to modulate CheA autophosphorylation (Lowenthal et al., 2009; Pittman et al., 2001). Future insights on TlpA/ TlpB and accessory protein arrangements will be necessary to clarify possible cooperative roles of these proteins in $\mathrm{H}$. pylori colonization.

TlpA seems to be a ubiquitously distributed protein among the Helicobacter sp., including H. hepaticus, H. mustelae, H. felis and other sixteen H. pylori strains (http://blast.ncbi. nlm.nih.gov/Blast.cgi). In addition, Andermann et al. (2002) have shown that tlpA loss-of-function impairs colonization capability of $H$. pylori. This evidence suggests a strong role of TlpA in H. pylori survival, inflammatory evasion and in re-population after antibiotic treatment, marking it a possible target for inhibitor drug design against this receptor and/or protein partners involved in TlpA signal transduction. Future

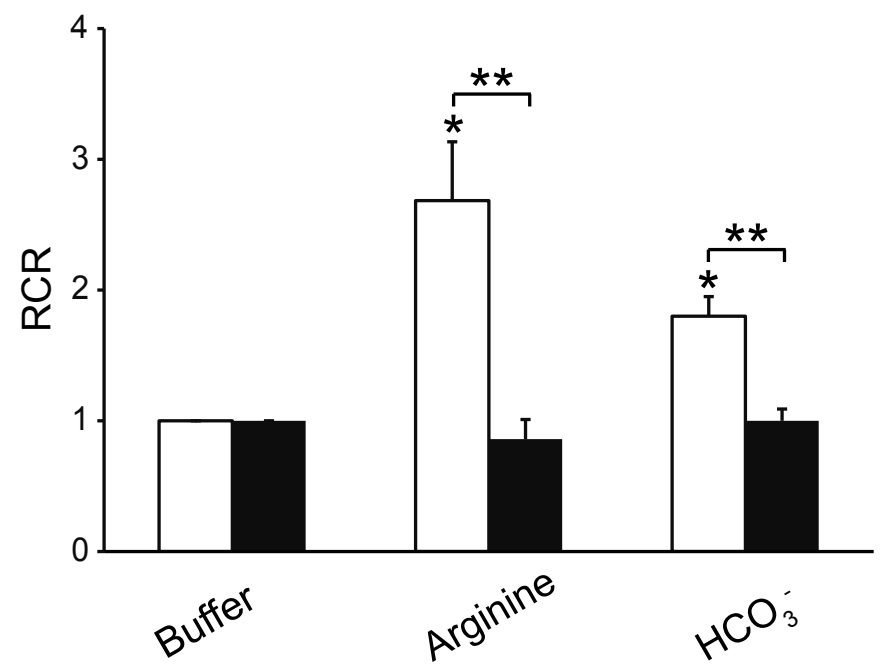

Figure 4. tIpA null mutant shows loss of arginine and sodium bicarbonate chemotactic response. Relative chemotactic responses (RCR) of H. pylori 700392 (empty bars) and 700tlpA::cat (filled bars) are shown. Chemotactic properties of the tlpA null strain differed significantly $\left({ }^{*} p<0.05,{ }^{* *} p<0.01\right)$ from the isogenic parent strain. Averages and means from at least 5 independent experiments are shown. research in this field will open opportunities for new H. pylori eradication therapies.

\section{ACKNOWLEDGMENTS}

We thank Mr. N. Villarroel for his valuable technical support. This research was supported by Grant FONDECYT \# 1085193.

\section{REFERENCES}

ADLER J (1973) A method for measuring chemotaxis and use of the method to determine optimum conditions for chemotaxis by Escherichia coli. J Gen Microbiol 74: 77-91.

ANDERMANN TM, CHEN Y, OTTEMANN KM (2002) Two predicted chemoreceptors of Helicobacter pylori promote stomach infection. Infect Immun 70: 5877-5881.

BREN A, EISENBACH M (2000) How signals are heard during bacterial chemotaxis: protein-protein interactions in sensory signal propagation. J Bacteriol 182:6865-6873.

CASTILLO AR, WOODRUFF AJ, CONNOLLY LE, SAUSE WE, OTTEMANN KM (2008) Recombination-based in vivo expression technology identifies Helicobacter pylori genes important for host colonization. Infect Immun 76:5632-5644.

CERDA O, RIVAS A, TOLEDO H (2003) Helicobacter pylori strain ATCC700392 encodes a methyl-accepting chemotaxis receptor protein (MCP) for arginine and sodium bicarbonate. FEMS Microbiol Letters 224:175-181.

CROXEN MA, SISSON G, MELANO R, HOFFMAN PS (2006) The Helicobacter pylori chemotaxis receptor TlpB (HP0103) is required for $\mathrm{pH}$ taxis and for colonization of the gastric mucosa. J Bacteriol 188:26562665.

ERNST PB, GOLD BD (2000) The disease spectrum of Helicobacter pylori: the immunopathogenesis of gastroduodenal ulcer and gastric cancer. Ann Rev Microbiol 54:615-640.

LOWENTHAL AC, SIMON C, FAIR AS, MEHMOOD K, TERRY K, ANASTASIA S, OTTEMANN KM (2009) A fixed-time diffusion analysis method determines that the three cheV genes of Helicobacter pylori differentially affect motility. Microbiology (Reading, England) 155:11811191

MAZUMDER R, PHELPS TJ, KRIEG NR, BENOIT RE (1999) Determining chemotactic responses by two subsurface microaerophiles using a simplified capillary assay method. J Microbiol Methods 37:255-263.

McGEE DJ, LANGFORD ML, WATSON EL, CARTER JE, CHEN YT, OTTEMANN KM (2005) Colonization and inflammation deficiencies in Mongolian gerbils infected by Helicobacter pylori chemotaxis mutants. Infect Immun 73:1820-1827.

McGOWAN CC, COVER TL, BLASER MJ (1996) Helicobacter pylori and gastric acid: biological and therapeutic implications. Gastroenterology 110:926-938.

MENDZ GL, HAZELL SL (1996) The urea cycle of Helicobacter pylori. Microbiology (Reading, England) 142:2959-2967.

MIZOTE T, YOSHIYAMA H, NAKAZAWA T (1997) Urease-independent chemotactic responses of Helicobacter pylori to urea, urease inhibitors, and sodium bicarbonate. Infect Immun 65:1519-1521.

MOULTON RC, MONTIE TC (1979) Chemotaxis by Pseudomonas aeruginosa. J Bacteriol 37:274-280.

OTTEMANN KM, LOWENTHAL AC (2002) Helicobacter pylori uses motility for initial colonization and to attain robust infection. Infect Immun 70:1984-1990.

OWEN RJ, BICKLEY J (1997) Isolation of H. pylori genomic DNA and restriction analysis. In: Methods in Molecular Medicine: Helicobacter pylori Protocols, pp. 81-88. Edited by C. L. Clayton and H. L. T. Mobley. Totowa, NJ: Humana Press Inc.

PITTMAN MS, GOODWIN M, KELLY DJ (2001) Chemotaxis in the human gastric pathogen Helicobacter pylori: different roles for CheW and the three $\mathrm{CheV}$ paralogues, and evidence for CheV2 phosphorylation. Microbiology (Reading, England) 147:2493-2504.

SCHILLING CH, COVERT MW, FAMILI I, CHURCH GM, EDWARDS JS, PALSSON BO (2002) Genome-scale metabolic model of Helicobacter pylori 26695. J Bacteriol 184:4582-4593.

SCHREIBER S, KONRADT M, GROLL C, SCHEID P, HANAUER G, WERLING HO, JOSENHANS C, SUERBAUM S (2004) The spatial orientation of Helicobacter pylori in the gastric mucus. Proc Natl Acad Sci USA 101:5024-5029. 
SCHREIBER S, SCHEID P (1997) Gastric mucus of the guinea pig: proton carrier and diffusion barrier. Am J Physiol Gastrointest Liver Physiol 272:G63-G70.

SCHREIBER S, STÜBEN M, JOSENHANS C, SCHEID P, SUERBAUM S (1999) In vivo distribution of Helicobacter felis in the gastric mucus of the mouse: experimental method and results. Infect Immun 67:5151-5156.

SCHWEINITZER T, MIZOTE T, ISHIKAWA N, DUDNIK A, INATSU S, SCHREIBER S, SUERBAUM S, AIZAWA S, JOSENHANS C (2008) Functional characterization and mutagenesis of the proposed behavioral sensor TlpD of Helicobacter pylori. J Bacteriol 190:3244-3255.

STUART-TILLEY A, SARDET C, POUYSSEFUR J, SCHWARTZ MA, BROWN D, ALPER SL (1994) Immunolocalization of anion exchanger AE2 and cation exchanger NHE-1 in distinct adjacent cells of gastric mucosa. Am J Physiol 266:C559-C568.

TERRY K, WILLIAMS SM, CONNOLLY L, OTTEMANN KM (2005) Chemotaxis plays multiple roles during Helicobacter pylori animal infection. Infect Immun 73:803-811.

TOMB JF, WHITE O, KERLAVAGE AR, CLAYTON RA, SUTTON GG, FLEISCHMANN RD, KETCHUM KA, KLENK HP, GILL S, DOUGHERTY BA, NELSON K, QUACKENBUSH J, ZHOU L, KIRKNESS EF, PETERSON S, LOFTUS B, RICHARDSON D, DODSON R, KHALAK HG, GLODEK A, MCKENNEY K, FITZEGERALD LM, LEE $\mathrm{N}$, ADAMS MD, HICKEY EK, BERG DE, GOCAYNE JD, UTTERBACK
TR, PETERSON JD, KELLEY JM, COTTON MD, WEIDMAN JM, FUJII C, BOWMAN C, WATTHEY L, WALLIN E, HAYES WS, BORODOVSKY M, KARP PD, SMITH HO, FRASER CM, VENTER JC. (1997) The complete genome sequence of the gastric pathogen Helicobacter pylori. Nature 388:539-547.

WANG Y, TAYLOR DE (1990) Chloramphenicol resistance in Campylobacter coli: nucleotide sequence, expression, and cloning vector construction Gene 94:23-28.

WILLIAMS SM, CHEN YT, ANDERMANN TM, CARTER JE, MCGEE DJ, OTTEMANN KM (2007) Helicobacter pylori chemotaxis modulates inflammation and bacterium-gastric epithelium interactions in infected mice. Infect Immun 75:3747-3757.

WORKU ML, KARIM QN, SPENCER J, SIDEBOTHAM RL (2004) Chemotactic response of Helicobacter pylori to human plasma and bile. J Med Microbiol 53:807-811.

WORKU ML, SIDEBOTHAM RL, WALKER MM, KESHAVARZ T, KARIM QN (1999) The relationship between Helicobacter pylori motility, morphology and phase of growth: implications for gastric colonization and pathology. Microbiology 145:2803-2811.

WUNDER C, CHURIN Y, WINAU F, WARNECKE D, VIETH M, LINDNER B, ZÄHRINGER U, MOLLENKOPF HJ, HEINZ E, MEYER TF (2006) Cholesterol glucosylation promotes immune evasion by Helicobacter pylori. Nat Med 12:1030-1038. 